\title{
ANALYSIS OF HOSPITAL CHARGES FOR HEART TRANSPLANTS IN THE US: AN EMPIRICAL COMPARISON ACROSS REGIONS AND YEARS
}

\author{
Jeffrey J. Fountain, Nova Southeastern University, Fort Lauderdale, FL, U.S.A. \\ Ravi R. Chinta, Nova Southeastern University, Fort Lauderdale, FL, U.S.A. \\ Hailey Jaramillo, Austin Peay State University, Clarksville, TN, U.S.A.
}

dx.doi.org/10.18374/JIFE-20-1.7

\begin{abstract}
Purpose: Empirically explain the variance of inflation Adjusted Total Hospital Charges for heart transplants in the United States by patient demographics, patient care in hospitals, and hospital characteristics, and suggest recommendations for lowering heart transplant costs.Method: Analyze the Agency for Healthcare Research and Quality (AHRQ) data on hospital in-patient data that is available annually in the Healthcare Cost and Utilization Project (HCUP) databases. Regression and correlation analyses were used to identify statistically significant variables that explain the wide variance in Adjusted Total Hospital Charges for heart transplants.Results: Findings showed that Total Hospital Charges for heart transplants overall, even after adjusting for inflation, increased from 2011 to 2016, as well as across subgroups based on the combination of region and year. Statistically significant variables that impacted the Inflation Adjusted Total Hospital Charges included Length of Stay, Mortality Risk, Discharged Status (Alive/Dead). Surprisingly patient demographics (Age, Gender, Income) were significant only in two regions (Northeast and the South) in 2011. Findings also revealed that the R-Squared decreased in all regions except for the Midwest region which implies that other independent variables (explanatory variables) not considered in this research model had an impact on the Inflation Adjusted Total Hospital Charges. Conclusion: This study utilized big data to help uncover regional differences and statistically significant variables explaining part of the variance in Adjusted Total Hospital Charges for heart transplant patients. The results show that the U.S. healthcare system could implement better cost reforms if hospitals that are efficient at treating specific procedures, transfering their knowledge to less efficient hospitals in an effort to reduce the variance in Total Hospital Charges patients receive for the same procedure in different parts of the country.
\end{abstract}

Keywords: Heart Transplant; Total Hospital Charges; Health Care Cost; DRG 001; DRG 002; Patient Demographics; Length of Stay; Mortality Risk 